\title{
BMJ Open Comparative efficacy and safety of oral or transdermal opioids in the treatment of knee or hip osteoarthritis: a systematic review and Bayesian network meta-analysis protocol
}

\author{
Jun Wang, ${ }^{1}$ Yin Wang, ${ }^{2}$ Hui Zhang, ${ }^{1}$ Ming Lu, ${ }^{1}$ Weilu Gao, ${ }^{1}$ Li Yin, ${ }^{1}$ Zongsheng Yin ${ }^{1}$
}

To cite: Wang J, Wang Y, Zhang $\mathrm{H}$, et al. Comparative efficacy and safety of oral or transdermal opioids in the treatment of knee or hip osteoarthritis: a systematic review and Bayesian network metaanalysis protocol. BMJ Open 2018;8:e022142. doi:10.1136/ bmjopen-2018-022142

- Prepublication history and additional material for this paper are available online. To view these files, please visit the journal online (http://dx.doi. org/10.1136/bmjopen-2018022142).

Received 7 February 2018 Revised 23 August 2018 Accepted 13 September 2018

A) Check for updates

(C) Author(s) (or their employer(s)) 2018. Re-use permitted under CC BY-NC. No commercial re-use. See rights and permissions. Published by BMJ.

${ }^{1}$ Department of Orthopaedics, The First Affiliated Hospital of Anhui Medical University, Anhui, China

${ }^{2}$ Department of Plastic Surgery, The Fourth Affiliated Hospital of Anhui Medical University, Anhui, China

Correspondence to Professor Zongsheng Yin; yinzsahgk@163.com

\section{ABSTRACT}

Introduction 0steoarthritis is a common

degenerative joint disease that eventually leads to disability and poor quality of life. The main symptoms are joint pain and mobility disorders. If the patient has severe pain or other analgesics are contraindicated, opioids may be a viable treatment option. To evaluate and compare the efficacy and safety of opioids in the treatment of knee or hip osteoarthritis, we will integrate direct and indirect evidence using a Bayesian network meta-analysis to establish hierarchies of these drugs.

Methods and analysis We will search the Medical Literature Analysis and Retrieval System Online, Excerpta Medica database, Cumulative Index to Nursing and Allied Health Literature, Cochrane Library, Web of Science and PsycINF0 databases as well as published and unpublished research in international registries and regulatory agency websites for osteoarthritis reports published prior to 5 January 2018. There will be no restrictions on the language. Randomised clinical trials that compare oral or transdermal opioids with other various opioids, placebo or no treatment for patients with knee or hip osteoarthritis will be included. The primary outcomes of efficacy will be pain and function. We will use pain and function scales to evaluate the main outcomes. The secondary outcomes of safety will be defined as the proportion of patients who have stopped treatment due to side effects. Pairwise metaanalyses and Bayesian network meta-analyses will be performed for all related outcome measures. We will conduct subgroup analyses and sensitivity analyses to assess the robustness of our findings. The Grading of Recommendations, Assessment, Development and Evaluations framework will be used to assess the quality of the evidence contributing to each network assessment.

Ethics and dissemination This study does not require formal ethical approval because individual patient data will not be included. The findings will be disseminated through peer-reviewed publications or conference presentations.

PROSPERO registration number CRD42018085503.
Strengths and limitations of this study

While previous conventional paired meta-analyses focused on direct comparisons between opioid analgesics and placebo for osteoarthritis, this Bayesian network meta-analysis will combine direct evidence with indirect evidence to assess the interrelationships between a wide range of opioid analgesics, placebo and no treatment in multiple treatment comparisons.

- Subgroup and sensitivity analyses will provide implications for clinically relevant questions for later research directions.

- This method synthesises the data comprehensively and provides a clinically useful summary that can guide the development of a clinical prescription system.

- The different routes of administration (oral or transdermal), durations and frequencies may cause considerable heterogeneity.

\section{INTRODUCTION}

Description of the condition

Osteoarthritis (OA), also known as degenerative arthritis or senile arthritis, is a degenerative disease. ${ }^{1}$ Increased obesity, age, trauma to joint areas, excessive manual labour and decreased muscle strength and joint stability are important risk factors for $\mathrm{OA}^{2-5}$ The main clinical manifestations of $\mathrm{OA}$ are chronic pain, joint instability, stiffness, joint deformity and reduced imaging of the joint space; these manifestations eventually lead to progressive disability and reduce patient quality of life. ${ }^{16}$ Worldwide, OA, particularly OA of the knee and hip joints, is one of the leading causes of disability among the older adults. ${ }^{78}$ Research has shown that around one-third of older adults have OA. ${ }^{9}$ This highly prevalent disease and the accompanying disability have terrible effects on individuals and society. The burden of OA is usually measured by direct 
and indirect economic costs, including less explicit intangibles such as pain and reduced quality of life. ${ }^{10}$

Cartilage destruction, subchondral bone remodelling and synovitis are the major pathological features of OA. Changes in the internal environment of various tissue structures within the joint cavity are the main causes of these pathological features and include anabolic and catabolic imbalance, especially an increase in articular cartilage catabolism leading to a decrease in the regeneration ability of cartilage. ${ }^{11} 12$ Previous studies have shown that many factors may interfere with chondrocyte homeostasis, including abnormal mechanical loading of proinflammatory mediators and oxidative stress. ${ }^{13} 14$ These mediators can cause inflammation which, in addition to promoting serious chondrocyte apoptosis and articular cartilage damage, can stimulate the sensory nerves in the synovium and surrounding tissues. This nerve stimulation leads to the peripheral and central sensitisation of the adjacent tissues which further leads to chronic pain. ${ }^{15}$

\section{Description of the intervention}

Pain is the most relevant symptom of $\mathrm{OA}$; as the degree of pain increases, patient mobility is decreased, and the degree of disability increases. ${ }^{16}{ }^{17}$ Because of pain and functional limitations, the quality of life of patients with $\mathrm{OA}$ is even worse than that of patients with gastrointestinal or chronic respiratory system disorders. ${ }^{18}$

Therefore, alleviating pain, preventing muscle atrophy and reducing joint deformity, stiffness and other complications are the main therapeutic targets of OA. ${ }^{19} 20$ Currently, the treatment modalities for OA include invasive surgery, non-drug therapy and drug therapy.

Invasive surgery includes intra-articular injections and surgery. Intra-articular injections of agents such as hyaluronic acid, corticosteroids, ozone and platelet-rich plasma are used for the treatment of $\mathrm{OA}$, and these treatments have been proven to be effective. ${ }^{21-24}$ Surgery mainly includes total hip and knee replacement which can improve health-related quality of life in the late stage of OA. ${ }^{25}{ }^{26}$ However, surgery is not the first choice of treatment for $\mathrm{OA}$ in clinical practice due to the limited lifespan of an artificial prosthesis. Furthermore, if a prosthesis fails, the patient may face a second revision operation, and the risk of failure in such operations is high due to the loss of bone mass. Therefore, joint surgery is often considered the ultimate treatment for OA. Non-drug therapy is important for reducing pain and improving the physiological function of patients with OA. ${ }^{27}$ Non-drug therapies include weight reduction, exercise, changes in lifestyle and other physical therapy measures designed to slow the progression of $\mathrm{OA} .^{28-30}$

Drugs for the treatment of $\mathrm{OA}$ pain primarily include non-steroidal anti-inflammatory drugs (NSAIDs), opioid drugs, paracetamol, capsaicin and duloxetine. ${ }^{31}$ Currently, the use of NSAIDs for the treatment of OA pain is preferred in the clinic. However, NSAID use may cause serious adverse cardiovascular, gastrointestinal and renal events. ${ }^{32-34}$ Opioids may be a viable alternative for patients who do not adequately respond to routine treatment and when other analgesics are contraindicated. ${ }^{35}$

\section{Why it is important to perform this review}

Several systematic reviews have investigated the effectiveness of the agents used to treat OA. ${ }^{1231}$ However, previous studies have considered only direct evidence from headto-head comparisons and did not aim to synthesise all the available evidence. As a result, determining the best treatment based on previous studies is often difficult. Indirect comparisons are usually required to establish a 'ranking' (occasionally referred to as a 'league table') of interventions. The Bayesian network meta-analysis method allows for the coinstantaneous comparison of multiple opioid drug interventions in a unitary analysis and ranks the interventions accordingly. This approach provides estimates of treatment differences and uses the heterogeneities and inconsistencies found in the tests to evaluate the uncertainties in the resultant estimates. Therefore, this approach is particularly useful in situations involving many different intervention measures. ${ }^{36}$

\section{Objectives}

To systematically review, compare in terms of efficacy and safety, and rank opioid analgesics for hip or knee OA.

\section{METHODS}

\section{Study design}

This protocol follows the Preferred Reporting Items for Systematic Reviews and Meta-Analyses Protocols (see online supplementary file 1 ). ${ }^{37}$

\section{Criteria for the included studies \\ Types of studies}

All randomised controlled trials comparing oral or transdermal opioid therapies with other opioids, placebos or no intervention in patients with knee or hip OA will be included. Trials published as abstracts only will be excluded. We will not apply limits based on the language of the publication.

\section{Types of participants}

Trials with mixed populations of patients with OA of the knee or hip must either report the results separately or must have included at least $75 \%$ of the patients in the relevant comparisons to be eligible for inclusion.

\section{Types of interventions}

Comparisons of oral or transdermal opioid drugs with any type of opioid drug, placebo or no intervention will be included. Trials comparing the same type of opioid at different therapeutic doses will be considered as a different node in the Bayesian network analysis. Consequently, the following comparisons are eligible: opioid versus opioid, placebo versus opioid and no intervention versus opioid. 


\begin{tabular}{lll}
\hline \multicolumn{2}{l}{ Table 1} & Hierarchy of osteoarthritis pain and function measurement scales \\
\hline Hierarchy & Pain measurement scales & Function measurement scales \\
\hline 1 & Global Pain Index & Global disability score \\
\hline 2 & Pain on walking & Walking disability \\
\hline 3 & WOMAC Osteoarthritis Index pain subscore & WOMAC disability subscore \\
\hline 4 & Composite pain scores other than WOMAC & Composite disability scores other than \\
& & WOMAC \\
\hline 6 & Pain on activities other than walking (such as stair climbing) & Disability other than walking \\
\hline 7 & Rest pain or pain during the night & WOMAC Global Scale \\
\hline 8 & WOMAC global algofunctional score & Lequesne Osteoarthritis Index global score \\
9 & Lequesne Osteoarthritis Index global score & Other algofunctional scale \\
\hline 10 & Other algofunctional scale & Participant's global assessment \\
11 & Participant's global assessment & Physician's global assessment \\
\hline
\end{tabular}

WOMAC, Western Ontario and McMaster Universities.

Types of outcome measures

\section{Primary outcomes}

The primary outcomes will include pain and function. If data from more than one pain or function scale are provided in a single trial, we will follow the method described in previous studies ${ }^{38}$ and extract data according to the hierarchy. The detailed scale hierarchy is presented in table 1 .

\section{Secondary outcomes}

To assess the safety of opioids, we will extract the proportion of participant withdrawals due to adverse events.

\section{Data sources and search strategy}

\section{Electronic searches}

We will search the Medical Literature Analysis and Retrieval System Online and Excerpta Medica database databases via the Ovid platform, the Cochrane Central Register of Controlled Trials database via the Cochrane Library and the Cumulative Index to Nursing and Allied Health Literature database via EBSCO. We will also search the Web of Science and PsycINFO databases. All databases will be searched from implementation to 5 January 2018 using a previously reported search strategy. ${ }^{10122931}$ For the strategies that will be used in this review, see online supplementary file 2 .

\section{Searching other resources}

International registries of published and unpublished articles and the websites of regulatory agencies will be searched in our review. These sources include the following: the WHO International Clinical Trials Registry Platform, clinicaltrials.gov, the University hospital Medical Information Network-Clinical Trials Registry, the American College of Rheumatology, the European League Against Rheumatism and the US Food and Drug Administration reports. No language limitations will be applied.

\section{Study selection}

Two independent reviewers (YW and HZ) will evaluate all relevant titles and abstracts. The reviewers will use uniform standards to independently extract key study parameters, and any disagreements will be resolved by the third review (JW). There will be no language restrictions. If multiple studies describe the same experiment, the study with the most relatively complete data will be used in the analyses.

\section{Data extraction and management}

Two review authors (YW and HZ) will extract the trial information independently via a single purpose-built electronic database. Any differences will be resolved by consensus or discussion with the third author (JW). The following information will be extracted:

- Patient characteristics (average age, gender, duration of symptoms and the type of joint affected).

- Details of the intervention, including the route of administration, dosage (different doses of the same drug will be divided into different nodes), and frequency of the drug therapies and the treatment duration.

- Types of measures used and pain-related or function-related outcomes.

- Type of adverse effects related to the outcome.

- Outcome data for each endpoint of interest.

- Duration of the follow-up.

- Trial design (including eligibility criteria of patients).

- Trial size.

- Publication status.

- The type and source of financial support.

We will use the results from intention-to-treat analyses whenever possible. ${ }^{40}$ If we cannot calculate the effect size, we will contact the study authors for additional data. Research from non-English language journals will be electronically translated before assessment. 


\section{Box 1 Assessment of the risk of bias in the six domains ${ }^{43}$}

1. Was there adequate sequence generation (selection bias)?

2. Was allocation adequately concealed (selection bias)?

3. Was knowledge of the allocated interventions adequately prevented during the study (detection bias)?

4. Were incomplete outcome data adequately addressed (attrition bias)?

5. Are reported of the study free of selective reporting (reporting bias)?

6. Was the study apparently free of other problems that could put it at a risk of bias?

\section{Assessment of the risk of bias in the included studies}

Two review authors (ML and LY) will independently use the risk of bias assessment tools generated by the Cochrane Collaboration. ${ }^{41}$ Disagreements will be resolved by negotiation. We will systematically evaluate bias across six domains ${ }^{42}$ as illustrated in box 1 . All included trials will be classified into the following categories: low risk, high risk and unclear. ${ }^{41}$

\section{DATA SYNTHESIS AND ANALYSIS \\ Measures of treatment effects \\ Relative treatment effects}

We will estimate continuous variables using the standardised mean difference (SMD) with $95 \%$ credible intervals (CrIs). For categorical outcomes, ORs with $95 \%$ CrIs will be calculated for the analyses. In the presence of minimally informative priors, CrIs can be understood similarly to CIs, and at the conventional statistical significance level, a two-sided $\mathrm{p}<0.05$ can be assumed if the 95\% CrIs do not include $0 .{ }^{43}$ If SDs are not provided, we will calculate them from the SEs, CIs or $p$ values using a method described in previous studies. ${ }^{39} 44$ If some necessary data are not available, we will use approximations as previously described. ${ }^{35}$ To visually explain the pooled effects, we will transform the effect sizes into differences on a $10 \mathrm{~cm}$ Visual Analogue Scale (VAS) based on a median pooled SD of $2.5 \mathrm{~cm}$, as found in large-scale OA trials that have used $10 \mathrm{~cm}$ VASs to assess pain. ${ }^{44}$ SMDs of -0.20 correspond to approximate differences in pain scores between the experimental and control groups of 0.5 on a $10 \mathrm{~cm}$ VAS, -0.50 of 1.25 on a $10 \mathrm{~cm}$ VAS and -0.80 of 2 on a $10 \mathrm{~cm}$ VAS. ${ }^{445}$ Additionally, we will compare the effects with a prespecified minimal clinically important difference based on the median pooled SD of 0.37 which has been used in recent studies of patients with OA and corresponds to $0.9 \mathrm{~cm}$ on a $10 \mathrm{~cm} \mathrm{VAS}{ }^{46-49}$ We will also transform the SMDs for function to a Western Ontario and McMaster Universities Osteoarthritis Index score based on a median pooled SD of 2.1 units as observed in large-scale OA trials. ${ }^{5051}$

\section{Relative treatment ranking}

Each intervention and each outcome will be systematically evaluated and ranked. We will determine a treatment hierarchy using the surface under the cumulative ranking curve and the mean ranks. ${ }^{52}$

\section{Data analysis}

First, we will conduct paired meta-analyses by synthesising the studies that compare interventions head-tohead using a random-effects model. ${ }^{53}$ Then, we will use a Bayesian network meta-analysis to compare the different classes of oral or transdermal opioid treatments based on the median of the posterior distribution. ${ }^{545}$ A Bayesian random-effects model will be used because this model completely retains the within-trial randomised treatment comparisons of each study while combining all available comparisons between treatments and accounting for multiple comparisons within a trial in cases with more than two treatment arms. ${ }^{556}$ The between-trial variance of the treatment effects ( $\tau^{2}$ will be estimated from the posterior distribution. Pooled estimates will be performed with Markov chain Monte Carlo methods. Convergence of the Markov chains will be considered to be achieved if the Gelman-Rubin diagnostic plots indicate that the widths of the pooled runs and individual runs stabilise around the same value, and their ratio is approximately one. ${ }^{57}$

The analyses will be performed using Stata V.14.0 software and WinBUGS (MRC Biostatistics Unit 2007, V.1.4.3; Cambridge, UK).

\section{Assessment of statistical heterogeneity}

We will use $\mathrm{I}^{2}$ statistics and $\mathrm{p}$ values to assess the statistical heterogeneity of each pairwise comparison. ${ }^{58}$ In the Bayesian meta-analysis, we will calculate the heterogeneity of the treatment effects estimated from the posterior median between-trial variance $\left(\tau^{2}\right)$. Global heterogeneity will be assessed using the $\mathrm{I}^{2}$ statistic.

\section{Assessment of statistical inconsistency}

We will evaluate the inconsistencies locally in the network using the loop-specific approach. ${ }^{59}$ The design-by-treatment interaction model will also be used to calculate the consistency throughout the entire network. ${ }^{59}$

\section{Subgroup analyses}

To explore the robustness of the results, we will include the characteristics of the trials as covariates in the Bayesian meta-analysis to assess the primary outcomes based on the clinical characteristics, risk of bias and trial size. A random-effects meta-regression model $^{60}$ will be used to determine whether the treatment effects are affected by the following factors: (1) treatment duration (short term $\leq 1$ month and long-term $>1$ month); (2) trial size (small-scale: allocated participants $\leq 200$, and largescale: allocated participants $>200$ ); (3) high methodological quality as defined by adequate concealment of the allocation (adequate vs inadequate or unclear); (4) adequate blinding of the patients (adequate vs inadequate or unclear); (5) intention-to-treat analysis (yes vs no or unclear); (6) source of funding (independent of the pharmaceutical industry or unclear vs no); (7) type of OA (hip only vs knee only vs mixed); (8) type of opioid 
(oral vs transdermal); and (9) type of trial (published vs unpublished).

\section{Sensitivity analyses}

We will perform sensitivity network meta-analyses for the primary outcomes by omitting unpublished trials and trials with inadequate or unclear allocation concealment.

\section{Other analyses}

The Grading of Recommendations, Assessment, Development and Evaluations framework, which characterises the quality of evidence based on the study limitations, publication bias, indirectness, imprecision and inconsistency in the primary outcomes, will be used to evaluate the quality of evidence in each network. ${ }^{61}$ Additionally, a comparison-adjusted funnel plot will be drawn to detect any major publication bias in the Bayesian network meta-analysis. ${ }^{62}$

\section{Ethics and dissemination}

This systematic review and Bayesian meta-analysis do not require formal ethical approval because individual patient data are not included. The results will provide a general review and evidence for the efficacy and safety of oral or transdermal opioids in the treatment of knee or hip OA. The findings will be disseminated through peer-reviewed publications or conference presentations. The basic protocol amendments will be recorded in the full review.

\section{Patient and public involvement}

No patients or the public participated in the study.

\section{DISCUSSION}

This systematic review and Bayesian network meta-analysis will provide an assessment of opioid therapies in patients with knee or hip OA. Currently, NSAIDs remain the first-line drugs for OA treatment. Whether opioids can be used as a routine treatment for knee or hip OA is controversial. One review compared the efficacy of NSAIDs and opioids in the treatment of knee OA and found that the efficacy is essentially the same. ${ }^{63}$ To date, no systematic review on opioids for OA has carried out a network meta-analysis to compare efficacy and safety across different opioid analgesics. Our results will rank the efficacy and safety of opioids in the treatment of OA which has not been included in previous studies. The conclusions of this study may be beneficial for patients with knee or hip OA, clinicians and policy-makers. We will perform subgroup analysis to explore whether our findings are consistent across subgroups and explore the sources of heterogeneity. The proposed systematic review and network meta-analysis may have some potential limitations. The different routes of administration (oral or transdermal), durations and frequencies may cause considerable heterogeneity. Another limitation may be differences in the quality of the included studies which will limit the ability of this work to reach high-confidence conclusions.
Acknowledgements We are grateful to American Journal Experts (AJE) for excellent language assistance.

Contributors JW conceived the review and wrote the first draft of the protocol. WG and $Z Y$ revised the protocol. $Y W$ and $H Z$ are responsible for the development of the search strategy and data extraction. ML and LY will be responsible for assessing bias and data synthesis and analysis. All the authors have approved the publication of the protocol.

Funding The authors have not declared a specific grant for this research from any funding agency in the public, commercial or not-for-profit sectors.

Competing interests None declared.

Patient consent Not required.

Provenance and peer review Not commissioned; externally peer reviewed.

Open access This is an open access article distributed in accordance with the Creative Commons Attribution Non Commercial (CC BY-NC 4.0) license, which permits others to distribute, remix, adapt, build upon this work non-commercially, and license their derivative works on different terms, provided the original work is properly cited, appropriate credit is given, any changes made indicated, and the use is non-commercial. See: http://creativecommons.org/licenses/by-nc/4.0/.

\section{REFERENCES}

1. Chen K, Yan Y, Li C, et al. Increased 15-lipoxygenase-1 expression in chondrocytes contributes to the pathogenesis of osteoarthritis. Cell Death Dis 2017;8:e3109.

2. Cooper C, Inskip H, Croft P, et al. Individual risk factors for hip osteoarthritis: obesity, hip injury, and physical activity. Am J Epidemiol 1998;147:516-22.

3. Blagojevic M, Jinks C, Jeffery A, et al. Risk factors for onset of osteoarthritis of the knee in older adults: a systematic review and meta-analysis. Osteoarthritis Cartilage 2010;18:24-33.

4. Jørgensen KT, Pedersen BV, Nielsen NM, et al. Socio-demographic factors, reproductive history and risk of osteoarthritis in a cohort of 4.6 million danish women and men. Osteoarthritis Cartilage 2011;19:1176-82.

5. Dekker J, van Dijk GM, Veenhof C. Risk factors for functional decline in osteoarthritis of the hip or knee. Curr Opin Rheumatol 2009;21:520-4.

6. Guilak F. Biomechanical factors in osteoarthritis. Best Pract Res Clin Rheumatol 2011;25:815-23.

7. Cross M, Smith E, Hoy D, et al. The global burden of hip and knee osteoarthritis: estimates from the global burden of disease 2010 study. Ann Rheum Dis 2014;73:1323-30.

8. Holden MA, Burke DL, Runhaar J, et al. Subgrouping and TargetEd Exercise pRogrammes for knee and hip OsteoArthritis (STEER OA): a systematic review update and individual participant data metaanalysis protocol. BMJ Open 2017;7:e018971.

9. Bijlsma JW, Knahr K. Strategies for the prevention and management of osteoarthritis of the hip and knee. Best Pract Res Clin Rheumatol 2007;21:59-76.

10. Hunter DJ, Schofield D, Callander E. The individual and socioeconomic impact of osteoarthritis. Nat Rev Rheumatol 2014;10:437-41.

11. Dunlop DD, Semanik P, Song J, et al. Risk factors for functional decline in older adults with arthritis. Arthritis Rheum 2005;52:1274-82.

12. Cepeda MS, Camargo F, Zea C, et al. Tramadol for osteoarthritis. The Cochrane Library 2006:CD005522.

13. Goldring MB, Otero M, Tsuchimochi K, et al. Defining the roles of inflammatory and anabolic cytokines in cartilage metabolism. Ann Rheum Dis 2008;67:iii75-82.

14. Hui W, Young DA, Rowan AD, et al. Oxidative changes and signalling pathways are pivotal in initiating age-related changes in articular cartilage. Ann Rheum Dis 2016;75:449-58.

15. Kean WF, Kean R, Buchanan WW. Osteoarthritis: symptoms, signs and source of pain. Inflammopharmacology 2004;12:3-31.

16. Bjordal JM, Ljunggren AE, Klovning A, et al. Non-steroidal antiinflammatory drugs, including cyclo-oxygenase-2 inhibitors, in osteoarthritic knee pain: meta-analysis of randomised placebo controlled trials. BMJ 2004;329:1317.

17. Dieppe PA, Lohmander LS. Pathogenesis and management of pain in osteoarthritis. The Lancet 2005;365:965-73.

18. Reginster JY. The prevalence and burden of arthritis. Rheumatology 2002;41:3-6.

19. Odding E, Valkenburg HA, Algra D, et al. Associations of radiological osteoarthritis of the hip and knee with locomotor disability in the Rotterdam study. Ann Rheum Dis 1998;57:203-8. 
20. Towheed TE, Judd MJ, Hochberg MC, et al. Acetaminophen for osteoarthritis. Cochrane Database Syst Rev 2003:CD004257.

21. Jevsevar DS, Brown GA, Jones DL, et al. The American Academy of Orthopaedic Surgeons evidence-based guideline on: treatment of osteoarthritis of the knee, 2nd edition. J Bone Joint Surg Am 2013;95:1885-6.

22. McAlindon TE, Bannuru RR, Sullivan MC, et al. OARSI guidelines for the non-surgical management of knee osteoarthritis. Osteoarthritis Cartilage 2014;22:363-88

23. Hashemi M, Jalili P, Mennati S, et al. The effects of prolotherapy with hypertonic dextrose versus prolozone (intraarticular ozone) in patients with knee osteoarthritis. Anesth Pain Med 2015:5:e27585.

24. Cugat R, Cuscó X, Seijas R, et al. Biologic enhancement of cartilage repair: the role of platelet-rich plasma and other commercially available growth factors. Arthroscopy 2015;31:777-83.

25. Emkey R, Rosenthal N, Wu SC, et al. Efficacy and safety of tramadol/ acetaminophen tablets (Ultracet) as add-on therapy for osteoarthritis pain in subjects receiving a COX-2 nonsteroidal antiinflammatory drug: a multicenter, randomized, double-blind, placebo-controlled trial. J Rheumatol 2004;31:150-6.

26. Towheed TE, Hochberg MC. Health-related quality of life after total hip replacement. Semin Arthritis Rheum 1996;26:483-91.

27. Zhang W, Moskowitz RW, Nuki G, et al. OARSI recommendations for the management of hip and knee osteoarthritis, Part II: OARSI evidence-based, expert consensus guidelines. Osteoarthritis Cartilage 2008;16:137-62.

28. Chodosh J, Morton SC, Mojica W, et al. Meta-analysis: chronic disease self-management programs for older adults. Ann Intern Med 2005;143:427-38.

29. Warsi A, LaValley MP, Wang PS, et al. Arthritis self-management education programs: a meta-analysis of the effect on pain and disability. Arthritis Rheum 2003;48:2207-13.

30. Roddy E, Zhang W, Doherty M. Aerobic walking or strengthening exercise for osteoarthritis of the knee? A systematic review. Ann Rheum Dis 2005;64:544-8.

31. da Costa BR, Nüesch E, Kasteler R, et al. Oral or transdermal opioids for osteoarthritis of the knee or hip. Cochrane Database Syst Rev 2014:CD003115.

32. Rashad S, Hemingway A, Rainsford K, et al. Effect of non-steroidal anti-inflammatory drugs on the course of osteoarthritis. The Lancet 1989;334:519-22.

33. Herman JH, Appel AM, Khosla RC, et al. The in vitro effect of select classes of nonsteroidal antiinflammatory drugs on normal cartilage metabolism. J Rheumatol 1986;13:1014-8.

34. Harifforoosh S, Jamali F. Renal adverse effects of nonsteroidal antiinflammatory drugs. Expert Opin Drug Saf 2009;8:669-81.

35. Avouac J, Gossec L, Dougados M. Efficacy and safety of opioids for osteoarthritis: a meta-analysis of randomized controlled trials. Osteoarthritis Cartilage 2007;15:957-65.

36. Caldwell DM, Ades AE, Higgins JP. Simultaneous comparison of multiple treatments: combining direct and indirect evidence. $B M J$ 2005;331:897-900.

37. Moher D, Shamseer L, Clarke M, et al. Preferred reporting items for systematic review and meta-analysis protocols (PRISMA-P) 2015 statement. Syst Rev 2015;4:1.

38. Jüni P, Reichenbach S, Dieppe P. Osteoarthritis: rational approach to treating the individual. Best Pract Res Clin Rheumatol 2006;20:721-40.

39. Reichenbach S, Sterchi R, Scherer M, et al. Meta-analysis: chondroitin for osteoarthritis of the knee or hip. Ann Intern Med 2007:146:580-90.

40. Nüesch E, Trelle S, Reichenbach S, et al. The effects of excluding patients from the analysis in randomised controlled trials: metaepidemiological study. BMJ 2009;339:b3244.

41. The Cochrane Collaboration. Chapter 8: Assessing risk of bias in included studies. In: Higgins J, Green S, eds. Cochrane handbook for systematic reviews of interventions. . Version 5.1.0, 2001:323. 42-6. (updated March 2011).
42. Palmer SC, Mavridis D, Navarese E, et al. Comparative efficacy and safety of blood pressure-lowering agents in adults with diabetes and kidney disease: a network meta-analysis. Lancet 2015;385:2047-56.

43. Zhou X, Qin B, Whittington C, et al. Comparative efficacy and tolerability of first-generation and newer-generation antidepressant medications for depressive disorders in children and adolescents: study protocol for a systematic review and network meta-analysis. BMJ Open 2015;5:e007768.

44. Follmann D, Elliott P, Suh I, et al. Variance imputation for overviews of clinical trials with continuous response. J Clin Epidemiol 1992;45:769-73.

45. Da Costa BR, Reichenbach S, Keller N, et al. Effectiveness of non-steroidal anti-inflammatory drugs for the treatment of pain in knee and hip osteoarthritis: a network meta-analysis. The Lancet 2017:390:e21-33.

46. Eberle E, Ottillinger B. Clinically relevant change and clinically relevant difference in knee osteoarthritis. Osteoarthritis Cartilage 1999;7:502-3.

47. Angst F, Aeschlimann A, Stucki G. Smallest detectable and minimal clinically important differences of rehabilitation intervention with their implications for required sample sizes using WOMAC and SF-36 quality of life measurement instruments in patients with osteoarthritis of the lower extremities. Arthritis Rheum 2001;45:384-91.

48. Angst F, Aeschlimann A, Michel BA, et al. Minimal clinically important rehabilitation effects in patients with osteoarthritis of the lower extremities. J Rheumatol 2002;29:131-8.

49. Salaffi F, Stancati A, Silvestri CA, et al. Minimal clinically important changes in chronic musculoskeletal pain intensity measured on a numerical rating scale. Eur J Pain 2004;8:283-91.

50. Bellamy N. Outcome measurement in osteoarthritis clinical trials. $J$ Rheumatol of rheumatology 1995;43:49-51.

51. Dickersin K, Scherer R, Lefebvre C. Identifying relevant studies for systematic reviews. BMJ 1994;309:1286.

52. Salanti G, Ades AE, loannidis JP. Graphical methods and numerical summaries for presenting results from multiple-treatment metaanalysis: an overview and tutorial. J Clin Epidemiol 2011;64:163-71.

53. DerSimonian R, Laird N. Meta-analysis in clinical trials. Control Clin Trials 1986;7:177-88

54. Salanti G, Higgins JP, Ades AE, et al. Evaluation of networks of randomized trials. Stat Methods Med Res 2008;17:279-301.

55. Lu G, Ades AE. Combination of direct and indirect evidence in mixed treatment comparisons. Stat Med 2004;23:3105-24.

56. Cooper NJ, Sutton AJ, Lu G, et al. Mixed comparison of stroke prevention treatments in individuals with nonrheumatic atrial fibrillation. Arch Intern Med 2006;166:1269-75.

57. Brooks SP, Gelman A. General methods for monitoring convergence of iterative simulations. J Comput Graph Statist 1998;7:434-55.

58. White IR, Barrett JK, Jackson D, et al. Consistency and inconsistency in network meta-analysis: model estimation using multivariate metaregression. Res Synth Methods 2012;3:111-25.

59. Higgins JP, Jackson D, Barrett JK, et al. Consistency and inconsistency in network meta-analysis: concepts and models for multi-arm studies. Res Synth Methods 2012;3:98-110.

60. Dias S, Sutton AJ, Welton NJ, et al. Evidence synthesis for decision making 3: heterogeneity-subgroups, meta-regression, bias, and biasadjustment. Med Decis Making 2013;33:618-40.

61. Abe H, Minatoguchi S, Ohashi $\mathrm{H}$, et al. Renoprotective effect of the addition of losartan to ongoing treatment with an angiotensin converting enzyme inhibitor in type-2 diabetic patients with nephropathy. Hypertens Res 2007:30:929-35.

62. Estacio RO, Coll JR, Tran ZV, et al. Effect of intensive blood pressure control with valsartan on urinary albumin excretion in normotensive patients with type 2 diabetes. Am J Hypertens 2006;19:1241-8.

63. Smith SR, Deshpande BR, Collins JE, et al. Comparative pain reduction of oral non-steroidal anti-inflammatory drugs and opioids for knee osteoarthritis: systematic analytic review. Osteoarthritis Cartilage 2016;24:962-72. 